\title{
An Analysis of the Delay and Jitter Performance of DBA Schemes for Differentiated Services in EPONs
}

\author{
Su-il Choi* \\ School of Electronics and Computer Engineering, Chonnam National University, \\ 300 Yongbong-dong, Buk-gu, Gwangju 500-757, Korea
}

(Received June 8, 2009 : revised August 10, 2009 : accepted August 10, 2009)

\begin{abstract}
An Ethernet passive optical network (EPON) is a low-cost, high-speed solution to the bottleneck problem of a broadband access network. This paper analyzes the delay and the jitter performance of dynamic bandwidth allocation (DBA) schemes for differentiated services in EPONs. Especially, the average packet delay and the delay jitter of the expedited forwarding (EF) traffic class are compared, with consideration as to whether a cyclic or an interleaved polling scheme is superior. This performance evaluation reveals that the cyclic polling based DBA scheme provides constant and predictable average packet delay and improved jitter performance for the EF traffic class without the influence of load variations.
\end{abstract}

Keywords: Ethernet passive optical network (EPON), Dynamic bandwidth allocation (DBA), Packet delay, Jitter

OCIS codes: (060.4250) Networks; (060.0060) Fiber optics and optical communications; (060.4510) Optical communication

\section{INTRODUCTION}

EPONs appear to be natural candidates for the nextgeneration of broadband access networks. An EPON is comprised of an optical line terminal (OLT) residing in the central office and multiple optical network units (ONUs) near subscribers' locations. In the downstream direction of an EPON, Ethernet frames are broadcast by the OLT and are selectively received by each ONU. In the upstream direction, however, all of the ONUs must contend for a shared capacity link; this requires an appropriate access protocol. In order to achieve statistical multiplexing in EPON architecture, a multipoint control protocol (MPCP) is developed and standardized by IEEE $802.3 \mathrm{ah}$. To date, various DBA algorithms to provide differentiated classes of service for EPONs have been proposed $[1-8,10]$.

This paper analyzes the performance, packet delay and delay jitter of the expedited forwarding $(\mathrm{EF})$ traffic class, of several DBA schemes for the differentiated classes of service in EPONs. Detailed performance evaluation shows that the cyclic polling based DBA

\footnotetext{
*Corresponding author: sichoi@chonnam.ac.kr
}

scheme is superior to interleaved polling based DBA schemes. For delay and delay variation sensitive traffic (e.g., voice traffic), the cyclic polling based DBA scheme gives constant and predictable packet delay and improved jitter performance. The rest of this paper is organized as follows. Section II presents several DBA schemes to provide quality of service (QoS) in EPONs. Section III gives a detailed analysis used to compare the performance of several DBA schemes. Final conclusions are covered in Section IV.

\section{DBA SCHEMES FOR QOS IN EPONS}

The interleaved polling scheme with adaptive cycle time (IPACT) [1] requires the OLT to poll every ONU and dynamically assign its bandwidth before transmission. This bandwidth is allocated according to the buffer occupancy status of the ONU. Any unrequested bandwidth will not be granted therefore the scheduling frame size is not fixed. When the IPACT is applied for differentiated services in EPONs there is a light-load penalty problem. To eliminate the light-load penalty problem a two-stage queue scheme and a constant bit rate (CBR) 
credit scheme are suggested [2].

The authors [3] have proposed a DBA scheme in which ONU nodes were partitioned into two groups, underloaded and overloaded, according to their minimum guaranteed transmission window sizes. Hence, total bandwidth saved from underloaded group is reallocated to overloaded ONUs to improve their efficiency. However, with this scheme the OLT still only focuses on how to satisfy bandwidth requests from different ONUs and does not provide a prior service to high-priority traffic.

A class-based bandwidth allocation scheme [4] collects REPORT messages from all ONUs before bandwidth allocation. The OLT assigns a fixed bandwidth to the EF traffic in all ONUs regardless of their dynamics. The drawback to this scheme is in the long report collection time; it does not end until reports are received from all the ONUs. The hybrid slot-size/rate (HSSR) [5] scheme classifies traffic into two priority classes. It also allocates a fixed amount of bandwidth to the highpriority class in order to minimize the delay and the jitter of packets. The major advantage of this scheme is that it can ensure that the high-priority traffic will always be served earlier than the lower priority types within each frame. However, the fixed bandwidth cannot always satisfy the instantaneous traffic demands.

A two-layer bandwidth allocation (TLBA) [6] scheme provides differentiated services based on MPCP. With this scheme, an ONU is allowed to report all of its instantaneous traffic load for each traffic class separately. The OLT first allocates the bandwidth for different traffic classes, then further distributes the bandwidth allocated to one class among all the requesting ONUs. Within the same class, all the ONUs share the bandwidth fairly, following the max-min policy. In this scheme, the OLT maintains a table to store the bandwidth demands from all the ONUs in an increasing order. The drawback is that the service order of the ONUs changes in every scheduling cycle, which could make the waiting time for the EF packet of each ONU change drastically.

Another class-based bandwidth allocation scheme is the dynamic bandwidth allocation with multiple services (DBAM) [7]. Instead of providing multiple services among ONUs and end users separately, the approach of DBAM is to incorporate both of them into the REPORT/GATE mechanism with class-based bandwidth allocation. DBAM employs class-based traffic prediction to take the frames arriving during the waiting time into account. Hence, the OLT serves all of the ONUs in a fixed round robin order to facilitate traffic prediction.

The interleaved polling scheme polls the next ONU before the transmission from the previous one has arrived. Therefore, the interleaved polling based DBA scheme uses a variable frame size to allocate bandwidth to all the ONUs. However, cyclic polling based band- width allocation (CPBA) [8] uses a fixed frame size (1 $\mathrm{ms}$ or $2 \mathrm{~ms}$ ) to allocate upstream bandwidth. This scheme provides a constant and predictable packet delay, and improves jitter performance for EF traffic without the influence of the offered ONU load variation. An application of CPBA is the IPTV channel package delivery architecture [9] for the EPONs. The CPBA scheme decreases the network layer channel zapping time and the ONU-based VLAN and IGMP snooping mechanisms provide delivery of differentiated IPTV channel packages in $10 \mathrm{Gbit} / \mathrm{s}$ EPONs.

To support the different QoS in the EPON, a novel dynamic scheduling algorithm, termed as hybrid granting protocol (HGP), is proposed [10]. This algorithm uses grant-before-report (GBR) scheme for the EF traffic class to minimize the packet delay and jitter for delay and delay-variation sensitive traffic. For assured forwarding (AF) and best effort (BE) traffic classes, HGP uses grant-after-report (GAR) scheme, i.e., the grant always follows the reported information that it is based upon.

\section{PERFORMANCE EVALUATION OF DBA SCHEMES}

In order to compare the performance of several DBA schemes, we conducted the simulation with a network model, developed using the $\mathrm{C}++$ program language, which included one OLT and 16 ONUs. The distance from the OLT to any ONU was fixed at $20 \mathrm{~km}$. The link rate between the OLT and the ONU was $1 \mathrm{~Gb} / \mathrm{s}$, and the rate from the end uses to the ONU was 100 $\mathrm{Mb} / \mathrm{s}$. The gap time was set at $1 \mu \mathrm{s}$.

In order to support differentiated classes of service with different packet delay and jitter requirements, we use three prioritized services: expedite forwarding (EF) has the highest priority used for strict delay sensitive services. This is typically a constant bit rate (CBR) voice transmission. Assured forwarding $(\mathrm{AF})$, with medium priority for services of nondelay sensitive variable bit rate (VBR) services such as a video stream. Finally, best effort (BE) with the lowest priority for delay tolerant services, which include web browsing, background file transfers and e-mail applications. As illustrated in Figure 1, DBA schemes use one of three types of scheduling frames: a variable scheduling frame, a fixed scheduling frame, and a two-layer scheduling frame.

Since EF service is narrowband, a T1 connection which consumes $4.48 \mathrm{Mbit} / \mathrm{s}$ of bandwidth was used. The remaining load was evenly distributed between the $\mathrm{AF}$ and BE services. To closely emulate the self-similar property of $\mathrm{AF}$ and $\mathrm{BE}$ traffic, we generated detailed self-similar traffic models using a Pareto distribution for all of the ONUs. To simplify the simulations, we also assumed that the total network load was evenly 


\begin{tabular}{|c|c|c|c|c|c|c|c|}
\hline$\longleftarrow$ & $k$-th sc & duling fram & $\longrightarrow$ & - & & -th scheduling & 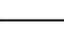 \\
\hline $\begin{array}{c}\text { ONU } \\
1\end{array}$ & ONU & $\cdots-\cdot$ & $\underset{\mathrm{N}}{\mathrm{ONU}}$ & $\begin{array}{c}\text { ONU } \\
1\end{array}$ & $\begin{array}{c}\text { ONU } \\
2\end{array}$ & & $\underset{\mathrm{N}}{\mathrm{ONU}}$ \\
\hline
\end{tabular}

(a) The variable DBA scheduling frame

\begin{tabular}{|c|c|c|c|c|c|c|c|}
\hline & \multicolumn{2}{|c|}{ k-th scheduling frame } & 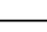 & E & \multicolumn{2}{|c|}{$(\mathrm{k}+1)$-th scheduling } & 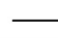 \\
\hline $\begin{array}{c}\text { ONU } \\
1\end{array}$ & $\begin{array}{c}\text { ONU } \\
2\end{array}$ & $\cdots \cdots$ & $\underset{\mathrm{N}}{\mathrm{ONU}}$ & $\begin{array}{c}\text { ONU } \\
1\end{array}$ & $\begin{array}{c}\text { ONU } \\
2\end{array}$ & & $\underset{\mathrm{N}}{\mathrm{ONU}}$ \\
\hline
\end{tabular}

(b) The fixed DBA scheduling frame

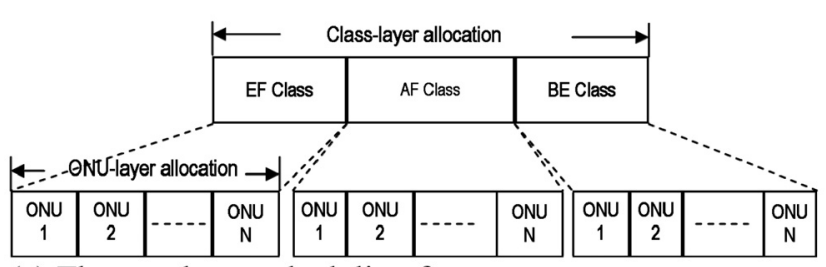

(c) The two-layer scheduling frame

FIG. 1. The DBA scheduling frames in EPONs.

distributed amongst all the ONUs and that the ONUs were equally weighted.

\section{The packet delay performance of EF service}

The packet delay is defined as the time between packet arrival and packet departure in the queue of an ONU. The average packet delay $d_{a v g}$ is obtained by:

$$
d_{\text {avg }} \approx \begin{cases}\frac{1}{2} T_{\text {poll }} & \text { one-stage queue } \\ \frac{3}{2} T_{\text {poll }} & \text { two-stage queue }\end{cases}
$$

where $T_{\text {poll }}$ is the variable or fixed polling time. Figure 2 compares the average packet delay of EF service for the IPACT with a two-stage queue, IPACT with CBR credit, Assi's DBA, TLBA, DBAM, and CPBA schemes. The DBA scheme based on variable scheduling frame size reduces the average EF packet delay as compared to a cyclic polling-based DBA scheme for light load scenario. At light loads, the polling cycle time of the interleaved polling scheme decreases to the round trip time (RTT), thus it reduces the average EF packet delay. However, it also reduces the capacity of downstream link to $94.624 \%$ of $1 \mathrm{~Gb} / \mathrm{s}$ for 16 ONUs and $89.248 \%$ of $1 \mathrm{~Gb} / \mathrm{s}$ for 32 ONUs, due to the control message exchanges between the OLT and the ONUs. At heavy load, IPACT with two-stage queue, Assi's DBA and TLBA schemes give an average EF packet delay of $3 \mathrm{~ms}$, and the IPACT with CBR credit, DBAM and CPBA schemes show the average EF packet delay as $1 \mathrm{~ms}$. The DBA scheme with two-stage queue shows three times the increase in average packet delay of EF traffic. This is because an EF packet that has arrived at a random time will wait half a cycle in the first stage queue and exactly one cycle in the second stage queue. This increased EF packet delay is the drawback of the two-stage queuing scheme.

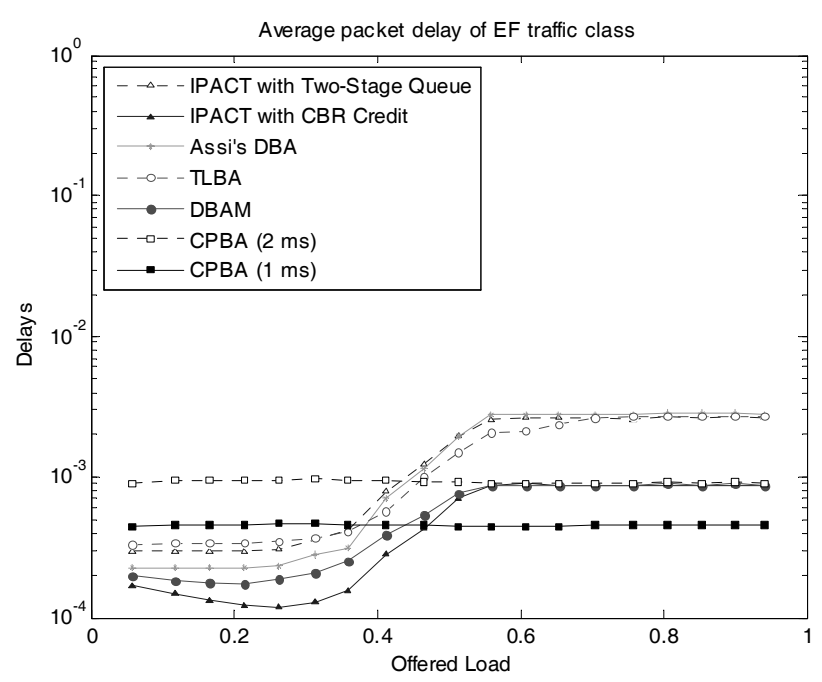

FIG. 2. The average packet delay of EF traffic class, utilizing several DBA schemes.

The advantage of CPBA is the constant and predictable average EF packet delay as shown by $T_{\text {poll }} / 2$ without the effect of the ONU load variation. When the scheduling frame size is set to $1 \mathrm{~ms}$ or $2 \mathrm{~ms}$, the CPBA scheme provides an average EF packet delay of $0.5 \mathrm{~ms}$ or $1 \mathrm{~ms}$.

\section{The jitter performance of EF service}

The packet delay variation, known as jitter, can be divided into two categories, intrawindow jitter and interwindow jitter [10]. Since EF traffic is non-bursty, it is reasonable to assume that the inter-arrival time of two successive EF packets is greater than the transmission time of the first EF packet as seen by the ONU. Hence the intrawindow jitter is defined as the packet delay variation of two consecutively departed EF packets from the same ONU in the same transmission window. The $j$-th delay jitter within the $i$-th window, $J_{i j}^{a}$, is obtained by:

$$
J_{i j}^{a}=d_{i j}-d_{i(j-1)}=T_{\text {trans }}-T_{\mathrm{int}} \equiv C
$$

where $d_{i j}$ is the $j$ th packet delay within the $i$-th window, $T_{\text {int }}$ and $T_{\text {trans }}$ are the interarrival time and packet transmission time respectively. In the same transmission window, the EF packet delay decreases by a constant rate, while its actual value depends on the delay value of the first departed EF packet in the transmission window, i.e., $d_{1}$.

The interwindow jitter is the variation of the first packet delay between two consecutive transmission windows. The interwindow jitter between the $i$-th window and $(i-1)$-th window, $J_{i}^{e}$, is formulated by: 


$$
J_{i}^{e}=d_{i 1}-d_{(i-1) 1}
$$

where $d_{i 1}$ is the first packet delay within the $i$-th window. The interwindow jitter consequently maps the distribution property of the total EF delay sequence for the ONU. EF packets with more fluctuation in their interwindow jitter tend to be continuously overdelayed or underdelayed, in respect to their mean value, and thus the total EF delay sequence appears to be more dispersed. Conversely, less fluctuation in the interwindow jitter tends to keep the EF packet delay distributed evenly around their mean value and the total EF delay sequence more centralized.

When the IPACT with CBR credit algorithm is applied for QoS in EPON, Figure 3 shows the probability density function (PDF) of the first departed EF service packet

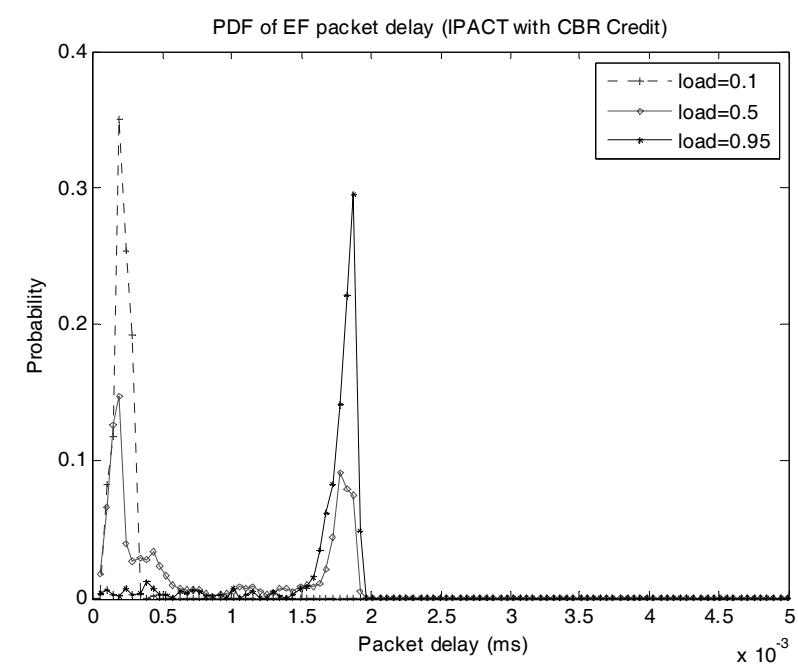

FIG. 3. The PDF of first departed EF packet delay using IPACT with CBR credit.

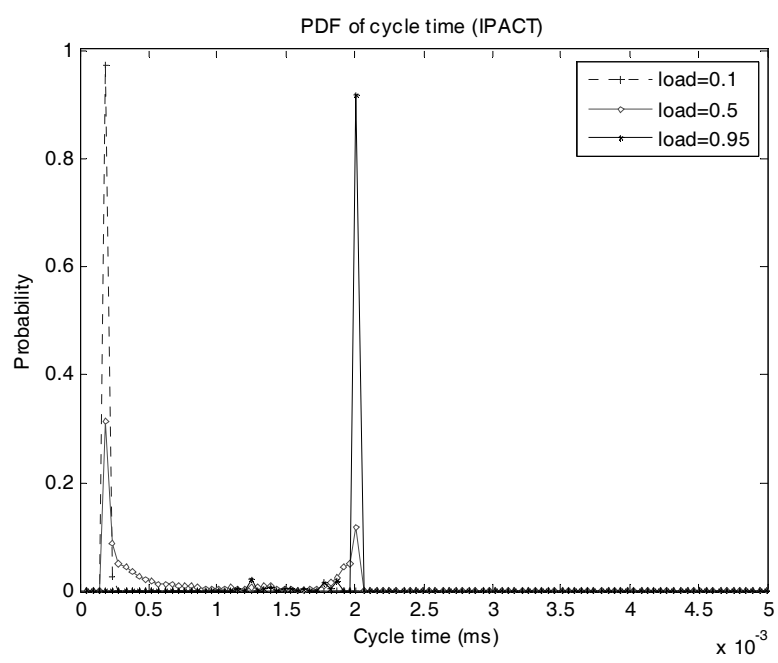

FIG. 4. The PDF of cycle time using IPACT with CBR credit. delay on all the ONUs at light (10\%), medium (50\%), and heavy loading $(95 \%)$ scenarios. It is shown that the first departed EF packet delay sequence centers at 0.17 $\mathrm{ms}$ and $1.75 \mathrm{~ms}$ for light load and heavy load scenarios, respectively. However, the PDF of the first departed EF packet delay sequence for medium loading scenario is dispersed and shows oscillation. This proves the jitter performance of variable polling based DBA schemes deteriorates in the medium load and variable load scenarios. The result of EF delay variation is due to the variable cycle time of the IPACT with CBR credit as shown in Figure 4.

Figure 5 shows the PDF of the first departed EF packet delay using the DBAM algorithm. The first departed EF packet delay sequence of DBAM centers at $0.36 \mathrm{~ms}$ and $1.85 \mathrm{~ms}$ for light load and heavy load scenarios respectively. It also shows a more deterio-

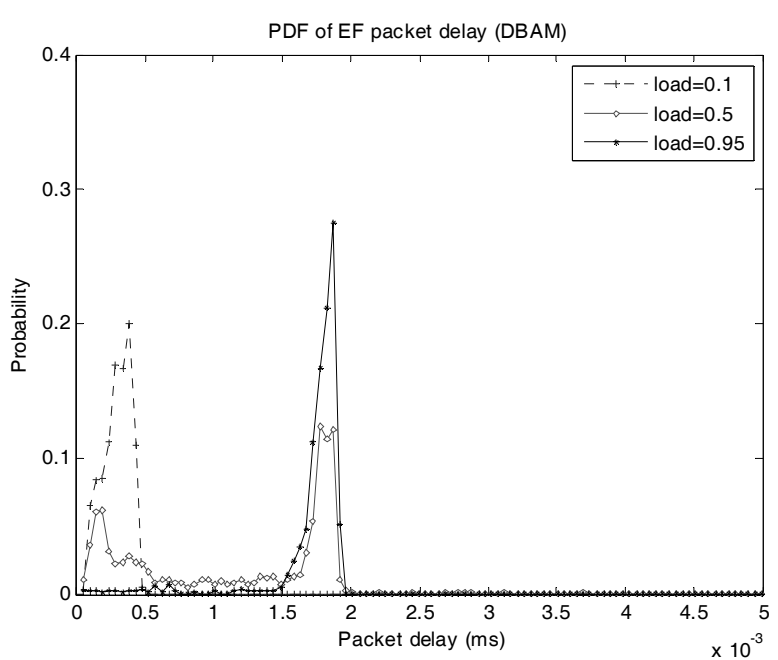

FIG. 5. The PDF of the first departed EF packet delay using DBAM.

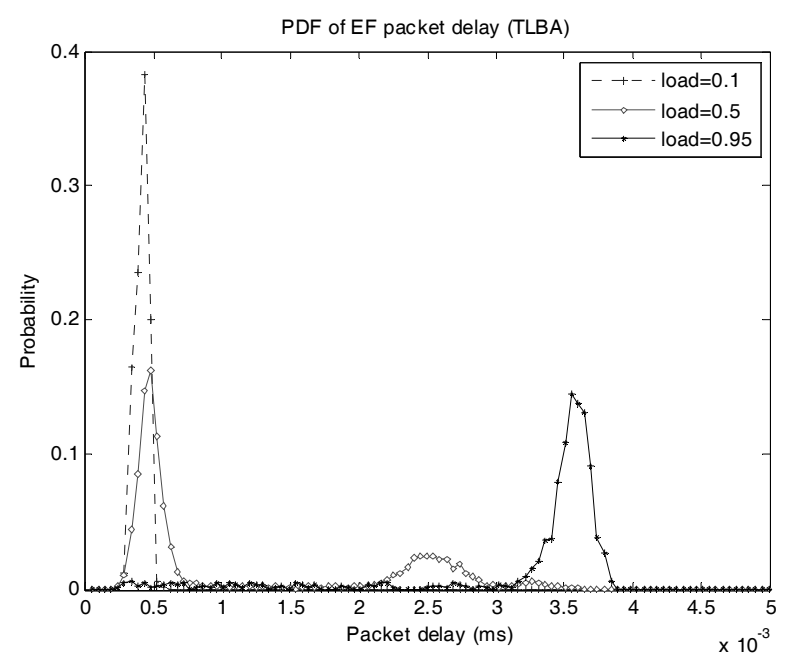

FIG. 6. The PDF of the first departed EF packet delay using TLBA. 


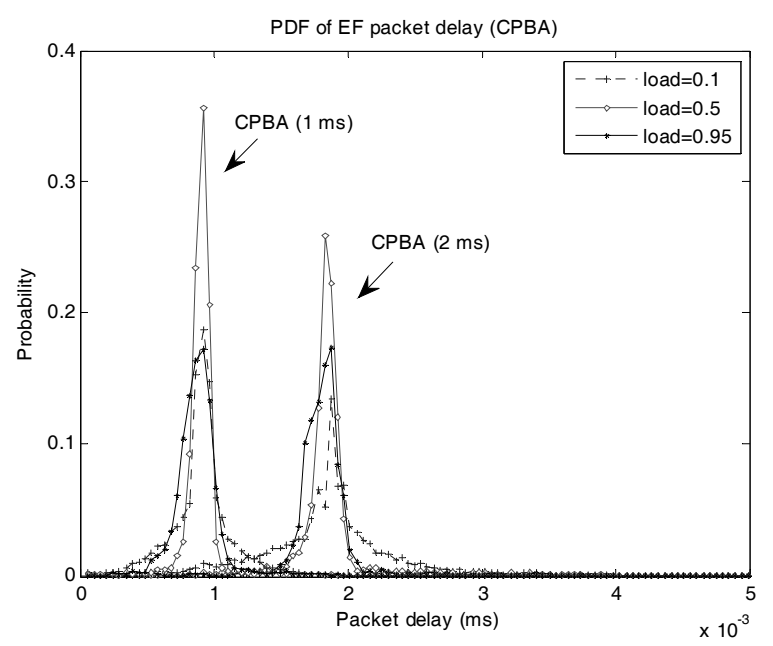

FIG. 7. The PDF of the first departed EF packet delay using CPBA (1 ms) and CPBA (2 ms).

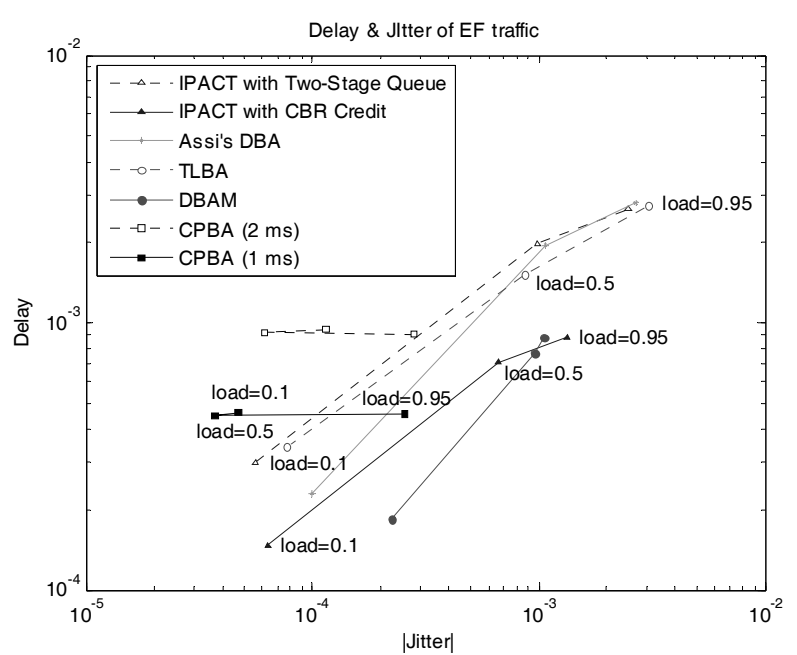

FIG. 8. A comparison of the average packet delay and interwindow jitter of the EF traffic class using several DBA schemes.

TABLE 1. The performance comparison of the EF traffic class using several DBA schemes

\begin{tabular}{|c|c|c|c|c|c|c|}
\hline \multirow[b]{2}{*}{ DBA Scheme } & \multicolumn{2}{|c|}{ Average packet delay $\left(d_{\text {avg }}\right)$} & \multicolumn{2}{|c|}{ Interwindow jitter $\left(\left|J^{e}\right|\right)$} & \multirow{2}{*}{$\begin{array}{l}\text { PDF of delay } \\
\text { (medium load) }\end{array}$} & \multirow[b]{2}{*}{ Complexity } \\
\hline & $\begin{array}{c}\text { Light load } \\
(0.1)\end{array}$ & $\begin{array}{c}\text { Heavy load } \\
(0.95)\end{array}$ & $\begin{array}{c}\text { Light load } \\
(0.1)\end{array}$ & $\begin{array}{c}\text { Heavy load } \\
(0.95)\end{array}$ & & \\
\hline $\begin{array}{c}\text { IPACT with } \\
\text { Two-Stage Queue }\end{array}$ & $0.30 \mathrm{~ms}$ & $2.65 \mathrm{~ms}$ & $0.06 \mathrm{~ms}$ & $2.47 \mathrm{~ms}$ & dispersed & complex \\
\hline $\begin{array}{l}\text { IPACT with } \\
\text { CBR Credit }\end{array}$ & $0.15 \mathrm{~ms}$ & $0.88 \mathrm{~ms}$ & $0.06 \mathrm{~ms}$ & $1.34 \mathrm{~ms}$ & dispersed & complex \\
\hline Assi's DBA & $0.23 \mathrm{~ms}$ & $2.80 \mathrm{~ms}$ & $0.01 \mathrm{~ms}$ & $2.68 \mathrm{~ms}$ & dispersed & medium \\
\hline TLBA & $0.34 \mathrm{~ms}$ & $2.73 \mathrm{~ms}$ & $0.08 \mathrm{~ms}$ & $3.07 \mathrm{~ms}$ & dispersed & medium \\
\hline DBAM & $0.20 \mathrm{~ms}$ & $0.88 \mathrm{~ms}$ & $0.23 \mathrm{~ms}$ & $1.06 \mathrm{~ms}$ & dispersed & medium \\
\hline CPBA (2ms) & $0.94 \mathrm{~ms}$ & $0.90 \mathrm{~ms}$ & $0.12 \mathrm{~ms}$ & $0.28 \mathrm{~ms}$ & centralized & simple \\
\hline CPBA (1ms) & $0.46 \mathrm{~ms}$ & $0.46 \mathrm{~ms}$ & $0.05 \mathrm{~ms}$ & $0.26 \mathrm{~ms}$ & centralized & simple \\
\hline
\end{tabular}

rated jitter performance than that of an IPACT with CBR credit at the medium load and variable load scenarios.

The PDF of the first departed EF service packet delay using the TLBA scheme is shown in Figure 6 . The delay sequence of TLBA centers at $0.43 \mathrm{~ms}$ and 3.55 ms for light load and heavy load scenarios, respectively. The medium loading scenario shows a more dispersed and oscillated delay distribution between $0.48 \mathrm{~ms}$ and $2.48 \mathrm{~ms}$. The TLBA scheme also shows longer delay and more deteriorated jitter performance than that of the IPACT with CBR credit or the DBAM scheme at the medium load or variable load scenario.

When a cycling polling based DBA algorithm (CPBA) is applied for simulation, Figure 7 shows the PDF of the first departed EF service packet delay of all the ONUs at light (10\%), medium (50\%), and heavy loading (95\%) scenarios. The EF packet delay sequence centers at $1.8 \sim 1.85 \mathrm{~ms}$ for the CPBA with $2 \mathrm{~ms}$ cycle time scheme, and at $0.91 \mathrm{~ms}$ for the CPBA with $1 \mathrm{~ms}$ cycle time scheme. Note that the load variation has no effect on the EF packet delay. The CPBA scheme provides a predictable EF packet delay and an improved jitter performance as compared to the interleaved polling based DBA scheme.

The average packet delay and the absolute value of the interwindow jitter of EF service packet using several DBA schemes is shown in Figure 8. With the load variation scenario, the interleaved polling based DBA schemes show deteriorated delay and jitter performance, whereas the cyclic polling based DBA scheme gives a constant and predictable delay with improved jitter performance. The CPBA scheme with a cycle time of $1 \mathrm{~ms}$ shows the best delay and jitter performance, and is suitable for the QoS in EPONs. For each DBA scheme, the average packet delay, the absolute value of interwindow jitter, the PDF of EF packet delay and the implementation complexity are compared in Table 1. 


\section{CONCLUSION}

In this paper, we analyzed the delay and jitter performance of the EF service packet using several DBA schemes in EPONs. The DBA scheme, based on a variable scheduling frame, shows good delay performance at light load scenario, but has dispersed and deteriorated jitter performance at medium load and load variation scenarios. A fixed scheduling frame based DBA scheme, however, provides a constant and predictable EF packet delay and shows more centralized distributions than the one using the interleaved polling based DBA scheme.

\section{ACKNOWLEDGMENT}

This work was supported by the Korea Research Foundation Grant funded by the Korean Government (MOEHRD, Basic Research Promotion Fund) (KRF2007-331-D00312).

\section{REFERENCES}

1. G. Kramer, B. Mukherjee, and G. Pesavento, "Interleaved polling with adaptive cycle time (IPACT): a dynamic bandwidth distribution scheme in an optical access network," Photonic Network Comm. 4, 89-107 (2002).
2. G. Kramer, B. Mukherjee, S. Dixit, Y. Ye, and R. Hirth, "Supporting differentiated classes of service in Ethernet passive optical networks," Journal of Optical Networking 1, 280-298 (2002).

3. C. M. Assi, Y. Ye, S. Dixit, and M. A. Ali, "Dynamic bandwidth allocation for quality-of-service over Ethernet PONs," IEEE Journal on Selected Areas in Communications 21, 1467-1477 (2003).

4. S. I. Choi and J. D. Huh, "Dynamic bandwidth allocation algorithm for multimedia services over Ethernet PONs," ETRI Journal 24, 465-468 (2002).

5. F. An, H. Bae, Y. Hsueh, M. Rogge, L. Kazovsky, and K. Kim, "A new media access control protocol guaranteeing fairness among users in Ethernet-based passive optical networks," in Proc. IEEE OFC (Atlanta, GA, USA, Mar. 2003), pp. 134-135.

6. J. Xie, S. Jiang, and Y. Jiang, "A dynamic bandwidth allocation scheme for differentiated services in EPONs," IEEE Communications Magazine 42, 32-39 (2004).

7. Y. Lou and N. Ansari, "Bandwidth allocation for multiservice access on EPONs," IEEE Communications Magazine 43, 16-21 (2005).

8. S. I. Choi, "Cyclic polling-based dynamic bandwidth allocation for differentiated classes of service in Ethernet passive optical networks," Photonic Network Comm. 7, 87-96 (2004).

9. S. I. Choi, "IPTV delivery architecture in 10G EPONs using ONU-based multicast emulation," J. Opt. Soc. Korea 12, 69-78 (2008).

10. A. Shami, X. Bai, C. Assi, and N. Ghani, "Jitter performance in Ethernet passive optical networks," J. Lightwave Technol. 23, 1745-1753 (2005). 